(2) Open Access Full Text Article

\title{
Image-guided intensity-modulated radiotherapy for refractory bilateral breast cancer in a patient with extensive cutaneous metastasis in the chest and abdominal walls
}

\author{
This article was published in the following Dove Press journal: \\ OncoTargets and Therapy \\ 23 May 2016 \\ Number of times this article has been viewed
}

\author{
Yueh-Feng Lu' \\ Yu-Chin Lin ${ }^{2}$ \\ Kuo-Hsin Chen ${ }^{3,4}$ \\ Pei-Wei Shueng' \\ Hsin-Pei Yeh' \\ Chen-Hsi Hsieh 1,5,6 \\ 'Division of Radiation Oncology, \\ Department of Radiology, ${ }^{2}$ Division \\ of Oncology and Hematology, \\ Department of Medicine, \\ ${ }^{3}$ Department of Surgery, Far Eastern \\ Memorial Hospital, New Taipei City, \\ ${ }^{4}$ Department of Electrical Engineering, \\ Yuan-Ze University, Taoyuan, \\ ${ }^{5}$ Department of Medicine, ${ }^{6}$ Institute \\ of Traditional Medicine, School \\ of Medicine, National Yang-Ming \\ University, Taipei, Taiwan
}

\begin{abstract}
Treatment for bilateral breast cancer with chest wall and abdominal skin invasion normally involves conventional radiotherapy (RT); however, conventional RT provides inadequate target volume coverage and excessive treatment of large volumes of normal tissue. Helical tomotherapy (HT) has the ability to deliver continuous craniocaudal irradiation that suppresses junction problems and provides good conformity of dose distribution. A 47-yearold female with stage IV bilateral breast cancer with chest wall and pectoralis major muscle invasion, lymphadenopathy, bilateral pleural effusion, and multiple bone metastases received chemotherapy and target therapy beginning in January 2014; 4 months after the initiation of chemotherapy, computed tomography revealed progression of chest and abdominal wall invasion. A total dose of 70.2 Gy was delivered to both breasts, the chest wall, the abdominal wall, and the bilateral supraclavicular nodal areas in 39 fractions via HT. The total planning target volume was $4,533.29 \mathrm{~cm}^{3}$. The percent of lung volume receiving at least $20 \mathrm{~Gy}$ (V20) was $28 \%$, $22 \%$, and $25 \%$ for the right lung, left lung, and whole lung, respectively. The mean dose to the heart was $8.6 \mathrm{~Gy}$. Follow-up computed tomography revealed complete response after the RT course. Grade 1 dysphagia, weight loss, grade 2 neutropenia, and grade 3 dermatitis were noted during the RT course. Pain score decreased from 6 to 1 . No cardiac, pulmonary, liver, or intestinal toxicity developed during treatment or follow-up. Concurrent HT with or without systemic treatment could be a safe salvage therapy for chemorefractory locally advanced breast cancer patients with extensive cutaneous metastasis.
\end{abstract}

Keywords: bilateral breast cancer, cutaneous metastasis, helical tomotherapy, palliative, toxicity

\section{Introduction}

Breast cancer is the second most common malignancy in females and the most common type of tumor associated with cutaneous metastasis. ${ }^{1}$ Metastasis to the skin can result from lymphatic embolization, hematogenous or contiguous dissemination, or as a result of direct implantation during surgical procedures. ${ }^{2,3}$ The incidence of cutaneous manifestation of breast cancer is $23.9 \%{ }^{4}$ and the most common sites of cutaneous manifestation are the chest wall and abdomen. ${ }^{5}$

Patients with cutaneous metastasis typically present with pruritus, erythema, pain, stinging sensation, macular rash, infection, and thickening or hardening of the chest wall. In the majority of cases where skin metastasis has occurred, the primary cancer is widespread and may be untreatable. ${ }^{6}$ In such cases of disseminated disease, the goals
Correspondence: Chen-Hsi Hsieh Division of Radiation Oncology, Department of Radiology, Far Eastern Memorial Hospital, 2I Sec 2, Nanya S Road, Banciao District, New Taipei City 220, Taiwan

Tel +886289667000 ext 1033

Fax +886289664367

Email chenciab@gmail.com 
of treatment are usually to delay disease progression; relieve symptoms, including keeping lesions clean and dry; and improve quality of life.

Trastuzumab is active and well tolerated as first-line treatment for females with metastatic breast cancer with human epithermal growth factor receptor 2 overexpression. ${ }^{7}$ Addition of trastuzumab to chemotherapy (anthracycline and cyclophosphamide, or paclitaxel) was associated with a longer time to disease progression, higher rate of objective response, lower rate of death at 1 year with a $20 \%$ reduction, and longer survival. ${ }^{8}$ For patients with inoperable breast cancer resistant to anthracycline-based chemotherapy, multidisciplinary locoregional management could provide an impressive 5-year survival rate. ${ }^{9}$

Radiotherapy (RT) is used traditionally as a modality for control of local metastases of breast cancer ${ }^{10}$ and provides survival benefit for breast cancer patients with synchronous metastases. ${ }^{11}$ Furthermore, in RT concurrent with trastuzumab $^{12}$ or paclitaxel, ${ }^{13}$ better response rates are expected. Recently, helical irradiation of the total skin has been shown to be an effective modality for the treatment of cutaneous T-cell lymphoma. ${ }^{14,15}$ Herein, we present a case of bilateral breast cancer with extensive cutaneous metastasis that was successfully and safely treated by helical tomotherapy (HT) concurrent with chemotherapy.

\section{Case description}

Left breast cancer was diagnosed in a 47-year-old female in 2007. The patient refused conventional treatment and instead received alternative medicine only. In 2013, follow-up computed tomography $(\mathrm{CT})$ revealed a huge $(>18 \mathrm{~cm})$ tumor in the left breast, a tumor in the contralateral breast, chest wall, and pectoralis major muscle invasion, lymphadenopathy, bilateral pleural effusion, and multiple bone metastases. Histopathological analysis of biopsy specimens revealed estrogen and progesterone receptor-negative, and human epidermal growth factor receptor 2-positive invasive ductal carcinoma. Extensive breast cancer, cT4cN3bM1 (American Joint Committee on Cancer, 7th Edition), was diagnosed. The patient received paclitaxel ( $\left.80 \mathrm{mg} / \mathrm{m}^{2}\right)$, trastuzumab ( $\left.8 \mathrm{mg} / \mathrm{kg}\right)$, and denosumab (120 mg) beginning in January 2014; however, 4 months after initiation of chemotherapy, follow-up CT revealed progressive disease with bilateral chest wall and abdominal wall invasion. The patient suffered from burning, warmth, pruritus, stinging, and painful sensation that came from lesion sites simultaneously. Skin biopsy was suggested but the patient refused.

A total dose of 70.2 Gy was delivered to breasts, the chest wall, the abdominal wall, and the supraclavicular nodal areas in 39 fractions via Tomotherapy ${ }^{\circledR}$ Hi-Art system (Tomotherapy ${ }^{\circledR}$;
Accuray Inc., Madison, WI, USA) with $6 \mathrm{MV}$ photon beam between June 2014 and September 2014. The total planning target volume (PTV) was 4,533.29 $\mathrm{cm}^{3}$. Mean lung dose and the percent volume receiving of lung $\geqq 20$ Gy dose (V20) were 16.3 Gy and $28 \%$ for the right lung, 15.5 Gy and 22\% for the left lung, and 15.9 Gy and $25 \%$ for the whole lung, respectively. The V5 was 55\% for both lungs. The mean dose and V30 to the heart were 8.6 Gy and 5\%, respectively. The mean dose to trachea, esophagus, stomach, liver, and intestine was 15.6, 6.6, 9.7, 7.1, and 6.0 Gy, respectively. The maximum dose to the spinal cord was $24.74 \mathrm{~Gy}$ (Table 1 ). The conformity index was 0.8 and the uniformity index was 1.1 .

After completion of HT with concomitant chemotherapy (paclitaxel and trastuzumab), the level of carcinoembryonic antigen decreased from 133 to $4.5 \mathrm{ng} / \mathrm{mL}$ and cancer antigen 15-3 decreased from 54.1 to $10.3 \mathrm{U} / \mathrm{mL}$. Grade 1 dysphagia, weight loss, grade 2 neutropenia, and grade 3 dermatitis were scored using the Common Terminology Criteria for Adverse Events (CTCAE, v4.0) during the RT course. No cardiac, pulmonary, liver, or intestine toxicity developed during treatment or follow-up. CT 4 months later revealed a marked reduction in the size of bilateral breast tumors and resolution of chest wall masses and nodular lesions, suggesting good response to treatment (Figures 1 and 2). The fungating wounds and erythematous changes of the chest and abdominal walls had been eradicated.

Approximately 5 months after the completion of HT and chemotherapy, a follow-up CT of the brain revealed metastatic lesions. The patient received whole-brain RT at a total dose of $30 \mathrm{~Gy}$ in ten fractions. However, she died due to disease progression, bilateral pleural effusion, and pulmonary

Table I The dosimetry results of organs at risk

\begin{tabular}{lll}
\hline Organs at risk & Dose-volume & \\
\hline Whole lung & Mean dose & $15.9 \mathrm{~Gy}$ \\
& V20 Gy & $25 \%$ \\
& V5 Gy & $55 \%$ \\
Right lung & Mean dose & $16.3 \mathrm{~Gy}$ \\
& V20 Gy & $28 \%$ \\
& V5 Gy & $55 \%$ \\
Left lung & Mean dose & $15.2 \mathrm{~Gy}$ \\
& V20 Gy & $22 \%$ \\
Heart & V5 Gy & $55 \%$ \\
& Mean dose & $8.6 \mathrm{~Gy}$ \\
Spinal cord & V30 Gy & $5 \%$ \\
Trachea & Maximum dose & $24.74 \mathrm{~Gy}$ \\
Esophagus & Mean dose & $15.6 \mathrm{~Gy}$ \\
Stomach & Mean dose & $6.6 \mathrm{~Gy}$ \\
Liver & Mean dose & $9.7 \mathrm{~Gy}$ \\
Intestine & Mean dose & $7.1 \mathrm{~Gy}$ \\
\hline
\end{tabular}

Note: $V_{x G y}$, percent volume receiving of an organ $\geq_{x}$ Gy dose. 



Figure I Tumor regression after HT.

Notes: (A) CT image acquired in the axial plane reveals infiltrating tumors in bilateral breasts, cutaneous metastasis, and axillary lymphadenopathy before HT. (B) CT scan 4 months after completion of HT reveals marked tumor regression.

Abbreviations: CT, computed tomography; HT, helical tomotherapy.

infection 8 months after completion of breast RT. The need for informed consent was waived by the Institutional Review Board of the Far Eastern Memorial Hospital (FEMH-IRB104164-C) and retrospective data were collected after receiving approval from the Institutional Review Board of the Far Eastern Memorial Hospital (FEMH-IRB-104164-C).

\section{Discussion}

Excluding melanoma, breast cancer is the most common cancer to metastasize to the skin. ${ }^{16}$ Median survival of patients with metastatic breast cancer ranges from 18 to 24 months. ${ }^{17}$ The goals of care are to prolong survival, delay disease progression, and improve quality of life. For a single, small, well-vascularized cutaneous metastatic lesion, surgical excision can be considered. Hormonal therapy, chemotherapy, monoclonal antibody therapy, electrochemotherapy, photodynamic therapy, topical arsenic trioxide, a cytostatic protein kinase $\mathrm{C}$ inhibitor, and antiangiogenesis agents are the options available for breast cancer patients with metastatic cutaneous lesions. ${ }^{6,18-20}$
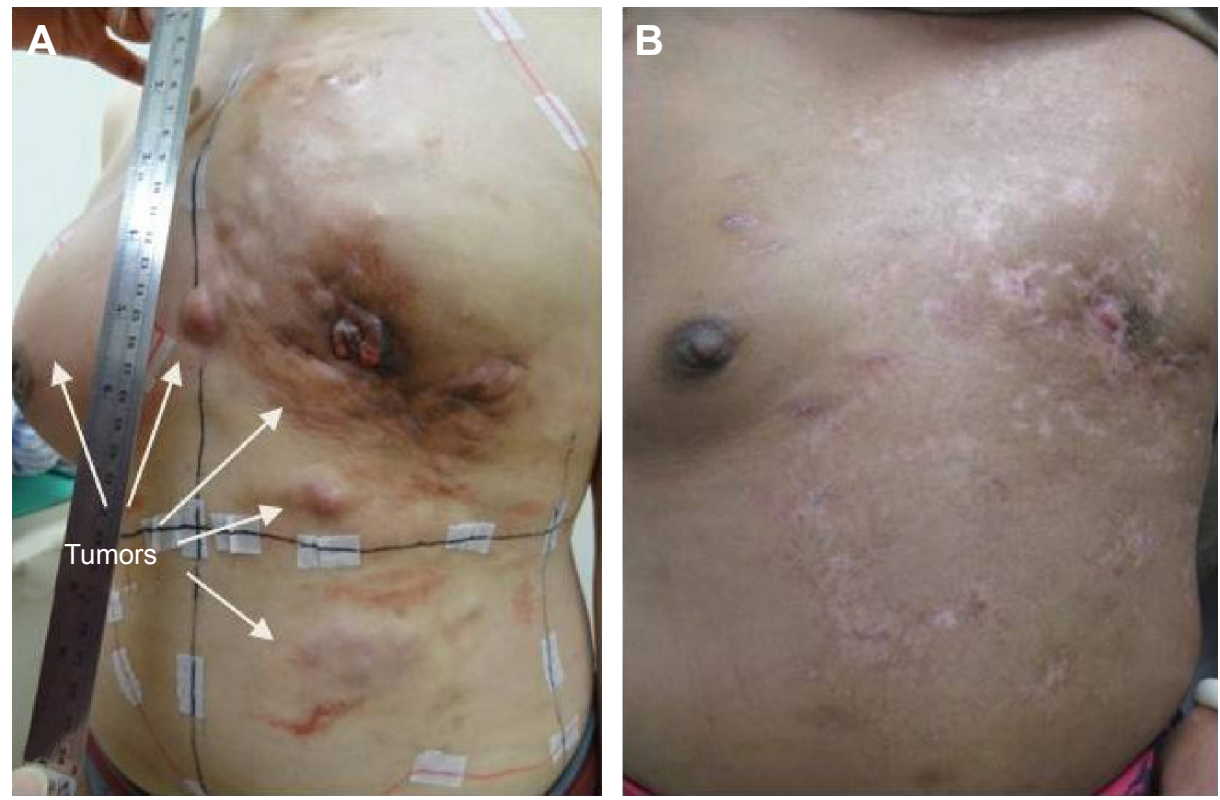

Figure 2 Cutaneous metastases regression after HT.

Notes: (A) Bilateral breast tumors with infiltrating cutaneous metastases in the chest and abdominal walls before HT. (B) Four months later, the skin was intact, with tumor regression in the chest and abdominal walls.

Abbreviation: HT, helical tomotherapy. 
Patients with stage IV disease, if able to undergo surgical excision of the primary tumor, achieve a superior survival rate than their nonsurgical counterparts. ${ }^{21}$ In breast cancer patients with inoperable locoregional disease because of primary disease extent after completion of primary chemotherapy, without evidence of distant metastases at diagnosis, the 5-year survival rate was $64 \%$ and alternative therapeutic strategies were suggested to this class of patients. ${ }^{9}$ In our patient, the lesions were located in the bilateral breasts, supraclavicular lymph nodes, and extensively on the chest and abdominal wall skin. The tumor volume maximum diameter was $32 \times 30 \mathrm{~cm}$ and the total volume was $4,533.29 \mathrm{~cm}^{3}$. She was not suitable for mastectomy because of the extensive lesions and hence alternative therapeutic strategies were considered.

RT is usually applied as a local treatment modality for symptomatic relief for breast cancer patients with limited cutaneous metastasis. However, studies have shown that locoregional treatment is associated with better survival. In a retrospective study on the impact of exclusive locoregional RT on survival, a total of 261 patients received no locoregional therapy, 249 received RT alone (with gross tumor volume boost to reach a total dose of 70-80 Gy), and 30 underwent surgery alone. Locoregional treatment include the group of RT alone and surgery alone. The overall survival of locoregional treatment group (RT and surgery) was $43.7 \%$; the overall survival of no locoregional treatment group was $26.7 \% .^{11}$

Our patient received extensive field irradiation with paclitaxel and trastuzumab and achieved a complete response of regional lesions without severe adverse effects. Adding trastuzumab to RT improved the efficacy with the rate of substantial pathologic response (complete response or microscopic residual disease) increasing to $43 \% .^{12}$ Furthermore, the pathological complete response rate for stage II and III breast cancer patients treated with concurrent paclitaxel and RT was 34\%. Also, such combination is effective and well tolerated. ${ }^{13}$ Putting these published observations together, it is suggested that trastuzumab and paclitaxel concurrent with RT could be one of the alternative therapeutic strategies for breast cancer patients who are diagnosed with extensive and inoperable locoregional disease.

Bilateral breast cancer with bilateral supraclavicular nodal area involvement and chest and abdominal wall metastasis is difficult to treat with conventional RT because of the extensive skin involvement, irregular surfaces, and proximity to critical organs. Treating shallow tumors with a homogeneous dose while delivering minimal doses to distal organs is challenging. ${ }^{22}$ Several factors can produce volumes of increased dose (hot spots) and decreased dose (cold spots), such as deviation of angle from normal, irregular surface, and internal heterogeneous tissues. ${ }^{23,24}$ Furthermore, field abutment to enlarge the radiation field can also create a cold spot upstream and a hot spot downstream of the region of intersection. Clearly, these unstable situations degrade the quality of the dose distribution and increase the failure rate of treatment.

HT, an image-guided intensity-modulated radiotherapy, is a treatment approach that combines with an on-board megavoltage CT imaging system. ${ }^{25}$ Image-guided intensitymodulated radiotherapy has the ability to correct setup errors, deliver continuous craniocaudal irradiation that suppresses junction problems, and provide good conformity of dose distribution. ${ }^{26}$ HT concurrent with mixed complete and directional block techniques can force the majority of the beamlets to be delivered to the PTV tangentially. This technique limits the depth of the dose distribution. Additionally, the mean doses for critical organs at risk are decreased successfully. ${ }^{14,15,27}$ The conformity index of HT in our patient was 0.81 and the uniformity index was 1.08 (Figure 3).

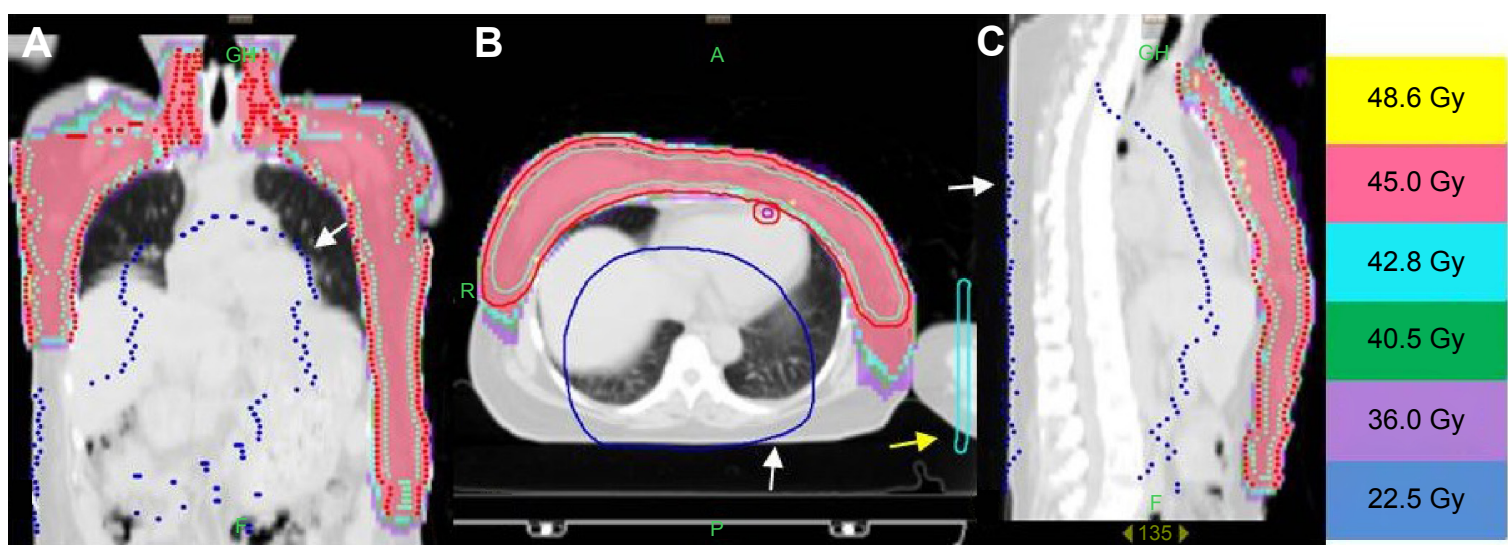

Figure 3 Planning target volume (red color line) delivered by helical tomotherapy with complete (the dark-blue area indicated by white arrows in panels $\mathbf{A}-\mathbf{C}$ ) and directional block (the light-blue area indicated by yellow arrow in panel B).

Notes: (A) Coronal view. (B) Axial view. (C) Sagittal view. 
V5 and V20 are the significant predictors of radiation pneumonitis (RP). Grade 3 pneumonitis is common when the V20 is $>32 \% .{ }^{28}$ Studies have shown that a V $20<25 \%$ of bilateral lungs can reduce the incidence of RP. ${ }^{29}$ Moreover, it is prudent to limit V20 to $\leq 30 \%-35 \%$ and the mean lung dose to $\leq 20-23 \mathrm{~Gy}$ (with conventional fractionation) if one wants to limit the risk of RP to $\leq 20 \%$ in definitive treatment of patients with non-small cell lung cancer. ${ }^{30}$ Furthermore, the cut-off value of ipsilateral and contralateral V5 under HT has been reported as $67.5 \%$ and $55.5 \%$, respectively. ${ }^{31}$ In this report, the V20 in the right, left, and whole lung was $28 \%, 22 \%$, and $25 \%$, respectively. Additionally, the V5 was $55 \%$ for both lungs. Mean lung doses were 16.3, 15.5, and 15.9 Gy in the right, left, and whole lung, respectively. Using the mixed block technique to limit the penetrating depth of skin by RT, HT successfully eradicated the tumor, relieved the symptoms, and kept the patient's critical organs safe.

Prevention of cardiovascular toxicity is another important issue for the treatment of breast cancer. The reported incidence of cardiac dysfunction ranges from 3\% to $7 \%$ in patients receiving trastuzumab alone and is as high as $13 \%$ in patients who receive concomitant paclitaxel and trastuzumab. ${ }^{32}$ In addition, rates of major coronary events have been reported to increase linearly with the mean dose to the heart, by $7.4 \%$ per gray. ${ }^{33}$ For partial irradiation, conservative (normal tissue complication probabilities) model-based estimates predict that a V25 Gy $<10 \%$ (in 2 Gy per fraction) is associated with a $<1 \%$ probability of cardiac mortality in 15 years after RT. ${ }^{34}$ Moreover, the risk of pericardial effusion is $13 \%$ with a V30 $<46$ Gy (or mean pericardial dose $<26$ Gy) vs $73 \%$ in patients with a V30 $>46$ Gy (or mean dose $>26$ Gy). ${ }^{35}$ The mean dose, $\mathrm{V} 25$, and V30 to the heart in our patient were $8.6 \mathrm{~Gy}, 8 \%$, and $5 \%$, respectively. The patient had received paclitaxel and trastuzumab concomitantly with HT; however, there was no evidence of ischemic heart disease after treatment. Therefore, the use of HT in the palliative setting might decrease the risk of developing RT-induced heart disease.

Concomitant chemotherapy, high radiation dose, and large PTV potentially increase the risk of developing grade 3 or higher acute skin toxicity. ${ }^{26}$ Because of the large tumor volumes and skin invasion in our patient, we delivered the radiation via bolus administration to ensure sufficient dose to the surface target volume and then boosted the dose to 70.2 Gy to gross tumors. The patient experienced grade 3 skin toxicity over the axilla and chest wall during the RT course. Treatment was interrupted for 1 week to allow the skin to heal, after which treatment continued with 45 and $61.20 \mathrm{~Gy}$. The patient completed the RT course uneventfully.
The ulcerated and fungating skin wounds healed completely after completion of RT and the visual analog scale pain score decreased from 6 to 1 .

\section{Conclusion}

The aims of care for breast cancer with extensive locoregional metastasis are to prolong survival, delay disease progression, and improve quality of life. HT with image-guided technique has the ability to correct setup errors, deliver continuous irradiation that suppresses junction problems, and provide good conformity and homogeneity of dose distribution to overcome irregular surface even under extensive tumor spreading. The combination of HT, target therapy, and chemotherapy was well tolerated and effective, which shed light on the possibility of alternative therapeutic approaches for locally advanced breast cancer patients with extensive cutaneous metastasis refractory to primary systemic therapy.

\section{Acknowledgments}

This work was supported by the Far Eastern Memorial Hospital grants (FEMH 104-2314-B-418-009-MY2). No funding was received for this report.

\section{Author contributions}

All authors contributed toward data analysis, drafting and revising the paper and agree to be accountable for all aspects of the work. All authors read and approved the final paper.

\section{Disclosure}

The authors report no conflicts of interest in this work.

\section{References}

1. Alcaraz I, Cerroni L, Rutten A, Kutzner H, Requena L. Cutaneous metastases from internal malignancies: a clinicopathologic and immunohistochemical review. Am J Dermatopathol. 2012;34(4): 347-393.

2. Oliveira GM, Zachetti DB, Barros HR, Tiengo A, Romiti N. Breast carcinoma en Cuirasse - case report. An Bras Dermatol. 2013;88(4): 608-610.

3. Sittart JA, Senise M. Cutaneous metastasis from internal carcinomas: a review of 45 years. An Bras Dermatol. 2013;88(4):541-544.

4. Lookingbill DP, Spangler N, Helm KF. Cutaneous metastases in patients with metastatic carcinoma: a retrospective study of 4020 patients. J Am Acad Dermatol. 1993;29(2 Pt 1):228-236.

5. De Giorgi V, Grazzini M, Alfaioli B, et al. Cutaneous manifestations of breast carcinoma. Dermatol Ther. 2010;23(6):581-589.

6. Moore S. Cutaneous metastatic breast cancer. Clin JOncol Nurs. 2002;6(5): 255-260.

7. Vogel CL, Cobleigh MA, Tripathy D, et al. Efficacy and safety of trastuzumab as a single agent in first-line treatment of HER2-overexpressing metastatic breast cancer. J Clin Oncol. 2002;20(3):719-726.

8. Slamon DJ, Leyland-Jones B, Shak S, et al. Use of chemotherapy plus a monoclonal antibody against HER2 for metastatic breast cancer that overexpresses HER2. N Engl J Med. 2001;344(11):783-792.

9. Huang E, McNeese MD, Strom EA, et al. Locoregional treatment outcomes for inoperable anthracycline-resistant breast cancer. Int J Radiat Oncol Biol Phys. 2002;53(5):1225-1233. 
10. Recht A, Come SE, Troyan SL, et al. Management of recurrent breast cancer. In: Harris IJ, Lippman M, Morrow M, Osborne C, editors. Diseases of the Breast. Philadelphia: Lippincott Williams and Wilkins; 2000: 731-748.

11. Le Scodan R, Stevens D, Brain E, et al. Breast cancer with synchronous metastases: survival impact of exclusive locoregional radiotherapy. J Clin Oncol. 2009;27(9):1375-1381.

12. Horton JK, Halle J, Ferraro M, et al. Radiosensitization of chemotherapyrefractory, locally advanced or locally recurrent breast cancer with trastuzumab: a phase II trial. Int J Radiat Oncol Biol Phys. 2010; 76(4):998-1004.

13. Chakravarthy AB, Kelley MC, McLaren B, et al. Neoadjuvant concurrent paclitaxel and radiation in stage II/III breast cancer. Clin Cancer Res. 2006;12:1570-1576.

14. Lin CT, Shiau AC, Tien HJ, Yeh HP, Shueng PW, Hsieh CH. An attempted substitute study of total skin electron therapy technique by using helical photon tomotherapy with helical irradiation of the total skin treatment: a phantom result. Biomed Res Int. 2013;2013:108794.

15. Hsieh CH, Shueng PW, Lin SC, et al. Helical irradiation of the total skin with dose painting to replace total skin electron beam therapy for therapy-refractory cutaneous CD4+ T-cell lymphoma. Biomed Res Int. 2013;2013:717589.

16. Mordenti C, Peris K, Fargnoli MC, Cerroni L, Chimenti S. Cutaneous metastatic breast carcinoma: a study of 164 patients. Acta Dermatoveneorol Alp Panonica Adriat. 2000;9(4):143-148.

17. Gennari A, Conte P, Rosso R, Orlandini C, Bruzzi P. Survival of metastatic breast carcinoma patients over a 20 -year period: a retrospective analysis based on individual patient data from six consecutive studies. Cancer. 2005;104(8):1742-1750.

18. Clive S, Gardiner J, Leonard RC. Miltefosine as a topical treatment for cutaneous metastases in breast carcinoma. Cancer Chemother Pharmacol. 1999;44 Suppl:S29-S30.

19. Schmidt G, Juhasz-Boss I, Solomayer EF, Herr D. Electrochemotherapy in breast cancer: A review of references. Geburtshilfe Frauenheilkd. 2014;74(6):557-562.

20. Lai YL, Chang HH, Huang MJ, et al. Combined effect of topical arsenic trioxide and radiation therapy on skin-infiltrating lesions of breast cancer - a pilot study. Anticancer Drugs. 2003;14(10):825-828.

21. Harris E, Barry M, Kell MR. Meta-analysis to determine if surgical resection of the primary tumour in the setting of stage IV breast cancer impacts on survival. Ann Surg Oncol. 2013;20(9):2828-2834.

22. Stewart JR, Leavitt DD, Prows J. Electron arc therapy of the chest wall for breast cancer: rationale, dosimetry, and clinical aspects. In: Vaeth JM, Meyer JL, editors. Frontiers of Radiation Therapy and Oncology Vol. 25: The Role of High Energy Electrons in the Treatment of Cancer. Basel: S. Karger AG; 1991:134-150.
23. Hogstrom KR, Fields RS. Use of CT in electron beam treatment planning: Current and future development. In: Ling CC, Rogers CC, Morton RJ, editors. Computerized Tomography in Radiation Therapy. New York: Raven Press; 1983:241-252.

24. Hogstrom KR. Dosimetry of electron heterogeneities. In: Wright A, Boyer A, editors. Advances in Radiation Therapy Treatment Planning, AAPM Monograph \#9. New York: American Institute of Physics; 1983: 223-243.

25. Mackie TR, Holmes T, Swerdloff S, et al. Tomotherapy: a new concept for the delivery of dynamic conformal radiotherapy. Med Phys. 1993;20: 1709-1719.

26. Chira C, Kirova YM, Liem X, et al. Helical tomotherapy for inoperable breast cancer: a new promising tool. Biomed Res Int. 2013;2013: 264306.

27. Shiau AC, Hsieh CH, Tien HJ. Left-sided whole breast irradiation with hybrid-IMRT and helical tomotherapy dosimetric comparison. Biomed Res Int. 2014;2014:741326.

28. Graham MV, Purdy JA, Emami B, et al. Clinical dose-volume histogram analysis for pneumonitis after 3D treatment for non-small cell lung cancer (NSCLC). Int J Radiat Oncol Biol Phys. 1999;45(2):323-329.

29. Zhang XJ, Sun JG, Sun J, et al. Prediction of radiation pneumonitis in lung cancer patients: a systematic review. J Cancer Res Clin Oncol. 2012; 138(12):2103-2116.

30. Marks LB, Bentzen SM, Deasy JO, et al. Radiation dose-volume effects in the lung. Int J Radiat Oncol Biol Phys. 2010;76(3 Suppl):S70-S76.

31. Kim Y, Hong SE, Kong M, Choi J. Predictive factors for radiation pneumonitis in lung cancer treated with helical tomotherapy. Cancer Res Treat. 2013;45(4):295-302.

32. Seidman A, Hudis C, Pierri MK, et al. Cardiac dysfunction in the trastuzumab clinical trials experience. J Clin Oncol. 2002;20(5): 1215-1221.

33. Darby SC, Ewertz M, McGale P, et al. Risk of ischemic heart disease in women after radiotherapy for breast cancer. N Engl J Med. 2013;368: 987-998.

34. Gagliardi G, Constine LS, Moiseenko V, et al. Radiation dosevolume effects in the heart. Int J Radiat Oncol Biol Phys. 2010; 76(3 Suppl):S77-S85.

35. Wei X, Liu HH, Tucker SL, et al. Risk factors for pericardial effusion in inoperable esophageal cancer patients treated with definitive chemoradiation therapy. Int J Radiat Oncol Biol Phys. 2008;70(3):707-714.
OncoTargets and Therapy

\section{Publish your work in this journal}

OncoTargets and Therapy is an international, peer-reviewed, open access journal focusing on the pathological basis of all cancers, potential targets for therapy and treatment protocols employed to improve the management of cancer patients. The journal also focuses on the impact of management programs and new therapeutic agents and protocols on

\section{Dovepress}

patient perspectives such as quality of life, adherence and satisfaction The manuscript management system is completely online and includes a very quick and fair peer-review system, which is all easy to use. Visit http://www.dovepress.com/testimonials.php to read real quotes from published authors. 